trial. The primary analysis of this trial did not, however, examine whether the delay in tumor progression conferred health-related qualityof-life (QoL) and symptom-control benefits. Subsequent analysis of the trial data by Siena et al. has highlighted an association between tumor-progression status and patient-reported QoL and symptomology.

In this study, health-related QoL and CRC symptomology were measured in 391 patients with refractory metastatic CRC who had been randomized to receive either panitumumab and best supportive care $(n=207)$ or best supportive care alone $(n=184)$. To determine the clinical significance of achieving stable disease in this population, the investigators conducted an analysis that excluded patients with an objective response to panitumumab. This investigation revealed that $80 \%$ of the treatment effect associated with panitumumab could be accounted for by patients without an objective response (i.e. patients with stable disease). In both treatment groups, patients who had achieved stable disease at week 8 reported significantly better control of disease-related symptoms than did those with disease progression. Lack of disease progression at 8 weeks' follow-up was associated with improved health-related QoL scores in panitumumab-treated patients.

The authors conclude that slowing disease progression in patients with metastatic CRC confers clinically meaningful improvements in symptom control and QoL.

Original article Siena S et al. (2007) Association of progression-free survival with patient-reported outcomes and survival: results from a randomised phase 3 trial of panitumumab. Br J Cancer 97: 1469-1474

\section{Time to biochemical failure predicts distant metastases and prostate-cancer-specific mortality}

Biochemical failure after initial treatment in patients with prostate cancer is an indicator that further treatment may be required, but deciding for whom and how quickly is sometimes problematic. In this study, Buyyounouski et al. investigated whether the interval to biochemical failure (IBF) might be a better predictor of clinical outcome than the American Society of Therapeutic Radiation Oncology (ASTRO) definition, and whether IBF could be used to predict for more-aggressive disease.
A total of 1,174 patients with prostate cancer treated by three-dimensional conformal radiotherapy only were examined, and 211 were identified as having biochemical failure (i.e. post-treatment prostate-specific antigen [PSA] level of $\geq P S A$ nadir plus $2 \mathrm{ng} / \mathrm{ml}$-the current ASTRO consensus definition). Statistical analysis revealed that an IBF of $<18$ months was an independent predictor of distant metastasis. Other independent predictors of distant metastasis included a Gleason score of 7-10, a PSA nadir of $\geq 2 \mathrm{ng} / \mathrm{ml}$, and decreasing radiation dose. In addition, multivariate analysis showed that an IBF of $<18$ months was an independent predictor of prostate-cancer-specific mortality.

The authors conclude that the IBF could be used to identify men at high risk of clinical failure and death. Those with an IBF of less than 18 months might benefit from aggressive salvage therapy or from participation in a clinical trial.

Original article Buyyounouski MK et al. (2007) Interval to biochemical failure highly prognostic for distant metastasis and prostate cancer-specific mortality after radiotherapy. Int J Radiat Oncol Biol Phys 70: 59-66

\section{Early detection of cervical lesions by use of human papillomavirus DNA testing}

High-risk types of human papillomavirus (HPV) are the main cause of cervical cancer, and HPV DNA tests are more sensitive in detecting cervical intraepithelial neoplasia grade 3 or higher (CIN3+) than are cytological smears. It is unknown, however, whether HPV DNA testing improves the effectiveness of cervical screening. Bulkmans et al. report the preliminary results from a population-based trial in The Netherlands that indicate that testing for HPV DNA as part of a primary screen may lead to earlier detection of clinically relevant cervical lesions.

Women aged 29-56 years, who participated in a regular cervical screening program, were randomly assigned to an intervention group (cytological smear and HPV DNA testing; $n=8,575$ ) or a control group (cytological testing only; $n=8,580$ ) for baseline screening. At a subsequent examination 5 years later, both groups were screened by cytological and HPV DNA testing.

At the initial screening, a higher number of $\mathrm{CIN} 3+$ lesions were detected in the intervention 